\title{
Derivation of male germ cells from bone marrow stem cells
}

\author{
Karim Nayernia ${ }^{1}$, Jae Ho Lee ${ }^{1}$, Nadja Drusenheimer ${ }^{1}$, Jessica Nolte ${ }^{1}$, Gerald Wulf ${ }^{2}$, \\ Ralf Dressel $^{3}$, Jörg Gromoll ${ }^{4}$ and Wolfgang Engel ${ }^{1}$ \\ ${ }^{1}$ Institute of Human Genetics, University of Göttingen, Göttingen, Germany; ${ }^{2}$ Department of Haematology and \\ Oncology; ${ }^{3}$ Department of Cellular and Molecular Immunology, University of Göttingen, Göttingen, Germany \\ and ${ }^{4}$ Institute of Reproductive Medicine, University of Münster, Münster, Germany
}

\begin{abstract}
Recent studies have demonstrated that somatic stem cells have a more flexible potential than expected, whether put into tissue or cultured under different conditions. Bone marrow (BM)-derived stem cells can transdifferentiate into multilineage cells, such as muscle of mesoderm, lung and liver of endoderm, and brain and skin of ectoderm origin. Here we show that BM stem cells are able to transdifferentiate into male germ cells. For derivation of male germ cells from adult BM stem (BMS) cells, we used the Stra8-enhanced green fluoresence protein (EGFP) transgenic mouse line expressing EGFP specifically in male germ cells. BMS cellderived germ cells expressed the known molecular markers of primordial germ cells, such as fragilis, stella, Rnf17, Mvh and Oct4; as well as molecular markers of spermatogonial stem cells and spermatogonia including Rbm, c-Kit, Tex18, Stra8, Piwil2, Dazl, Hsp90 $\alpha, \beta 1$ - and $\alpha 6$-integrins. Our ability to derive male germ cells from BMS cells reveals novel aspects of germ cell development and opens the possibilities for use of these cells in reproductive medicine.
\end{abstract}

Laboratory Investigation (2006) 86, 654-663. doi:10.1038/labinvest.3700429; published online 1 May 2006

Keywords: mesenchymal stem cells; transdifferentiation; spermatogonial stem cells

Male germ cells are derived from a founder population of primordial germ cells (PGC) that are set aside early in embryogenesis. ${ }^{1,2}$ PGC arise from the proximal epiblast, a region of the early mouse embryo that also contributes to the first blood lineages of the embryonic yolk sac. ${ }^{1}$ PGCs migrate through the dorsal mesentery and enter the developing fetal gonad, the genital ridge, between E10.5 and E12.5. Once they arrive in the genital ridge, the PGCs are enclosed by somatic Sertoli cells and become gonocytes. ${ }^{3}$ The gonocytes proliferate for a few days and then arrest in $\mathrm{G}_{0} / \mathrm{G}_{1}$ phase until birth. Within a few days after birth, the gonocytes resume proliferation to initiate spermatogenesis. By day 6 postpartum, these cells migrate to the basement membrane of seminiferous tubules and become undifferentiated type A spermatogonia, the spermatogonial stem cells (SSCs). SSCs normally divide asymmetrically, giving rise to one stem cell and one spermatogonia, which initiates differentiation to

Correspondence: Professor Dr K Nayernia, $\mathrm{PhD}$, Institute of Human Genetics, University of Göttingen, Heinrich Düker Weg 12, Göttingen 37073, Germany.

E-mail: knayern@gwdg.de

Received 13 October 2005; revised 27 February 2006; accepted 8 March 2006; published online 1 May 2006 produce spermatozoa. ${ }^{4,5}$ In the different stages of male germ cell differentiation, several molecular markers were identified, such as fragilis, ${ }^{6}$ stella, ${ }^{6}$ Rnf17, ${ }^{7}$ mouse vasa homolog (Mvh) ${ }^{8}$ and Oct ${ }^{9}$ for PGCs; and Rbm, ${ }^{10}$ c-Kit, ${ }^{11}$ Tex18, ${ }^{7}$ Stra8,${ }^{12}$ Piwil2, ${ }^{13}$ Dazl,${ }^{14}$ Hsp90 $\alpha,{ }^{15} \beta 1$ - and $\alpha 6$-integrins ${ }^{16}$ for spermatogonia and SSCs.

An area of research that generates great optimism is the use of stem cells for treatment of human diseases. Much of the excitement centers on embryonic stem cells (ES), but this approach remains controversial for ethical reasons; moreover, routine clinical application of this strategy is many years away. ${ }^{17,18}$ By contrast, bone marrow (BM) stem (BMS) cells from adult BM are well characterized and have long been used therapeutically. ${ }^{17}$ Accumulated evidence suggests that in addition to haematopoietic stem cells hematopoeitic stem cells, BM also harbors endothelial stem cells (ESC), mesenchymal stem cells (MSC), and multipotential adult progenitor cells. Adult bone-marrow-derived MSC are capable of differentiation along several tissue-forming cells such as bone, cartilage, fat, muscle, tendon, liver, kidney, heart, and even brain cells. ${ }^{19,20}$

In present study, we used the transgenic mouse line Stra8-enhanced green fluoresence protein 
(EGFP), expressing EGFP specifically in male germline stem cells, ${ }^{21}$ to isolate for the first time male germline stem cells from MSC.

\section{Materials and methods}

\section{Mouse Strain}

The transgenic mouse line used for isolation of BM was described previously. ${ }^{21}$

\section{Isolation of BMS Cells}

BM cells were collected by flushing the femurs from 8 to 12-week-old male Stra8-EGFP transgenic mice with phosphate-buffered saline (PBS) supplemented with $2 \%$ fetal bovine serum (Gibco, Paisley, UK). Cells were plated in murine mesenchymal stem cell medium, MesenCult, with murine mesenchymal stem cell stimulatory supplements (Stem Cell Technologies, Vancouver, Canada), further supplemented with $100 \mathrm{IU} / \mathrm{ml}$ penicillin and $100 \mu \mathrm{g} / \mathrm{ml}$ streptomycin (Gibco). Nonadherent cells were eliminated by a half medium change at day 3 and the whole medium was replaced weekly with fresh medium. The cells were grown for 2-3 weeks until attaining almost confluency. The whole adherent fraction was then detached by trypsinization and replated using a 1:3 dilution factor.

\section{Isolation of EGFP-Positive Cells from Testis}

Cell suspensions were prepared from testis of Stra8-EGFP transgenic mice according to published protocol. ${ }^{5}$ EGFP-positive cells, which contain SSCs, were sorted by fluorescence-activated cell sorting (FACS) as previously described. ${ }^{21}$

\section{Fluorescence-Activated Cell Sorting}

BM cells were grown in murine mesenchymal stem cell medium at a density of approximately $1-2 \times 10^{4}$ cells per $\mathrm{cm}^{2}$. Nonadherent cells were removed after 3-4 days and medium was replaced. Cultures were maintained in the above medium for an additional 2 weeks and then medium was changed by above medium containing retinoic acid (RA) (Sigma) at a final concentration of $10^{-6} \mathrm{M}$ for different times. Approximately $3 \%$ of all cells showed GFP expression $24 \mathrm{~h}$ after RA treatment. Positive cells were
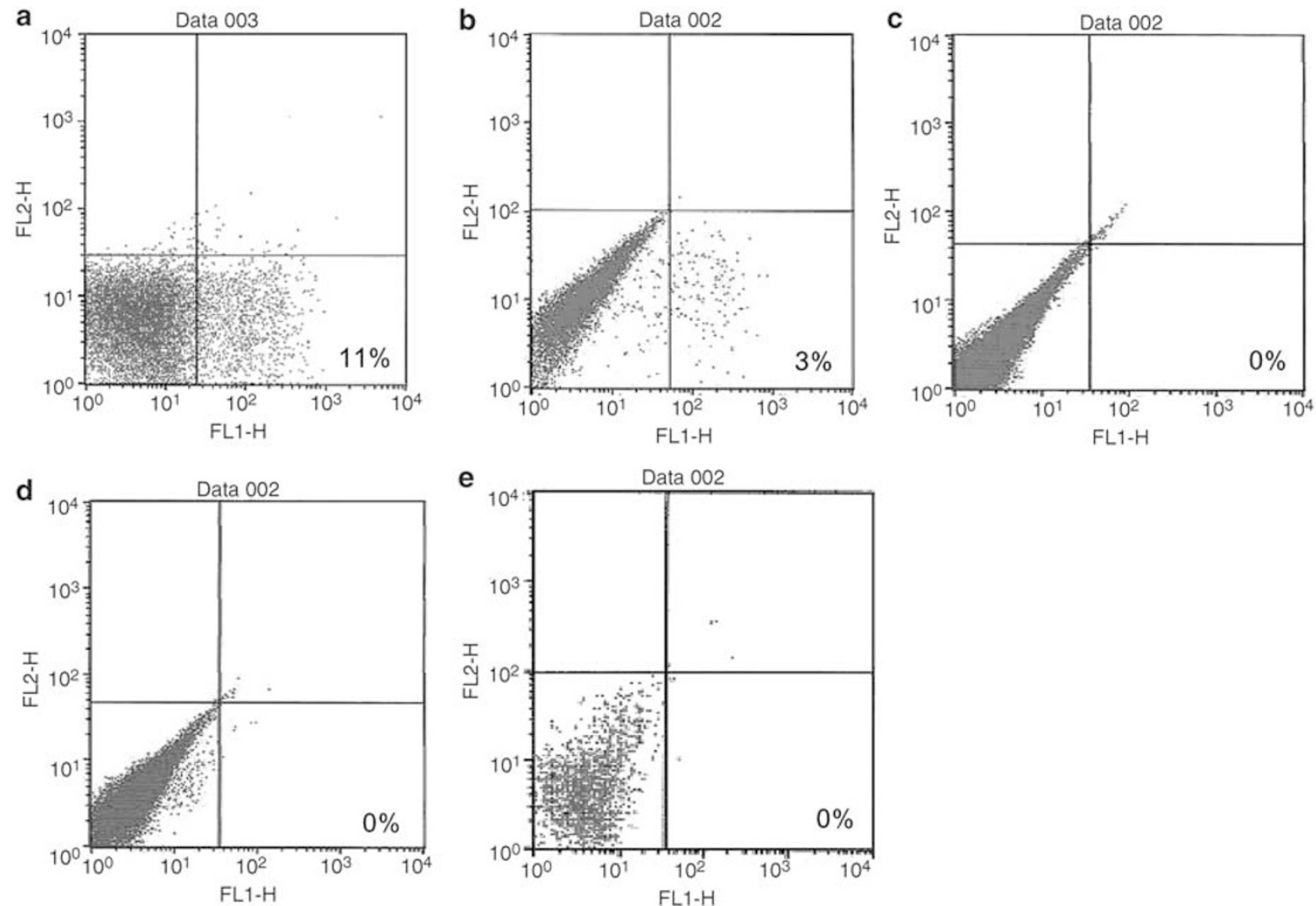

Figure 1 Fluorescence-activated cell sorting (FACS) analysis of cells isolated from Stra8-EGFP transgenic mice. (a) About 11\% of cells isolated from testis were EGFP positive without retinoic acid treatment. (b) FACS analysis of adherent bone cells cultured under conditions for mesenchymal stem cells present 3\% EGFP-positive cells after $24 \mathrm{~h}$ RA treatment. (c) FACS analysis of bone marrow stem cells without RA treatment, no positive cells could be isolated. (d and e) Fibroblast (d) and hematopoetic stem (e) cells show no EGFP-positive cells after RA treatment. 
sorted by FACS as previously described. Briefly, cells were dissociated with $0.25 \%$ trypsin/EDTA, neutralized with DMEM with 10\% FCS, washed twice with PBS and then resuspended in PBS containing $0.5 \%$ bovine serum albumin (BSA). Approximately $2 \times 10^{6}$ cells $/ \mathrm{ml}$ in PBS/BSA were used for sorting. The flow cytometry was performed on a FACStar Plus (Becton Dickinson) equipped with dual 488-nm argon and 633-nm helium neon lasers.

\section{RT-PCR and Immunohistochemical Analysis}

RNA isolation, RT-PCR and immunohistochemical analyses were performed as described previously. ${ }^{21}$ For confocal microscopical analysis, cells were grown for 21 days and EGFP-positive cell colonies were analyzed using a CarlZeiss confocal microscope.

\section{Transplantation and Analysis of Transplanted Testes}

BSC-derived germ cells were transplanted in seminiferous tubuli of busulfan-treated mice. Cell concentration for transplantation was $5-10 \times 10^{6} \mathrm{cells} / \mathrm{ml}$. Approximately $10 \mu \mathrm{l}$ of cell suspension was trans- planted as described previously. Testis cell suspension was prepared from Stra8-EGFP males and EGFP-positive cells were transplanted. As control, busulfan-treated nontransplanted males were used. After 8-12 months, recipient mice were killed and testes were examined by immunhistochemistry and RT-PCR analysis. ${ }^{21}$

\section{Results}

In addition to playing a fundamental role in very diverse processes such as vision and the growth and differentiation of numerous types of cells, vitamin A (retinol) and its principal biologically active derivative, RA, are clearly involved in the regulation of testicular functions in rodents. An excess of vitamin A leads to testicular lesions and spermatogenetic disorders, and a deficiency induces early cessation of spermatogenesis and adversely affects testosterone secretion. Here, we report that adherent BMS cells are able to differentiate to male germ cells by RA. We isolated and cultured these cells from transgenic mouse line Stra8-EGFP-expressing EGFP specifically in male germline stem cells. ${ }^{21}$ These cells $(11 \%)$ could be isolated from testicular cell suspension using fluorescence-activated cell sorting
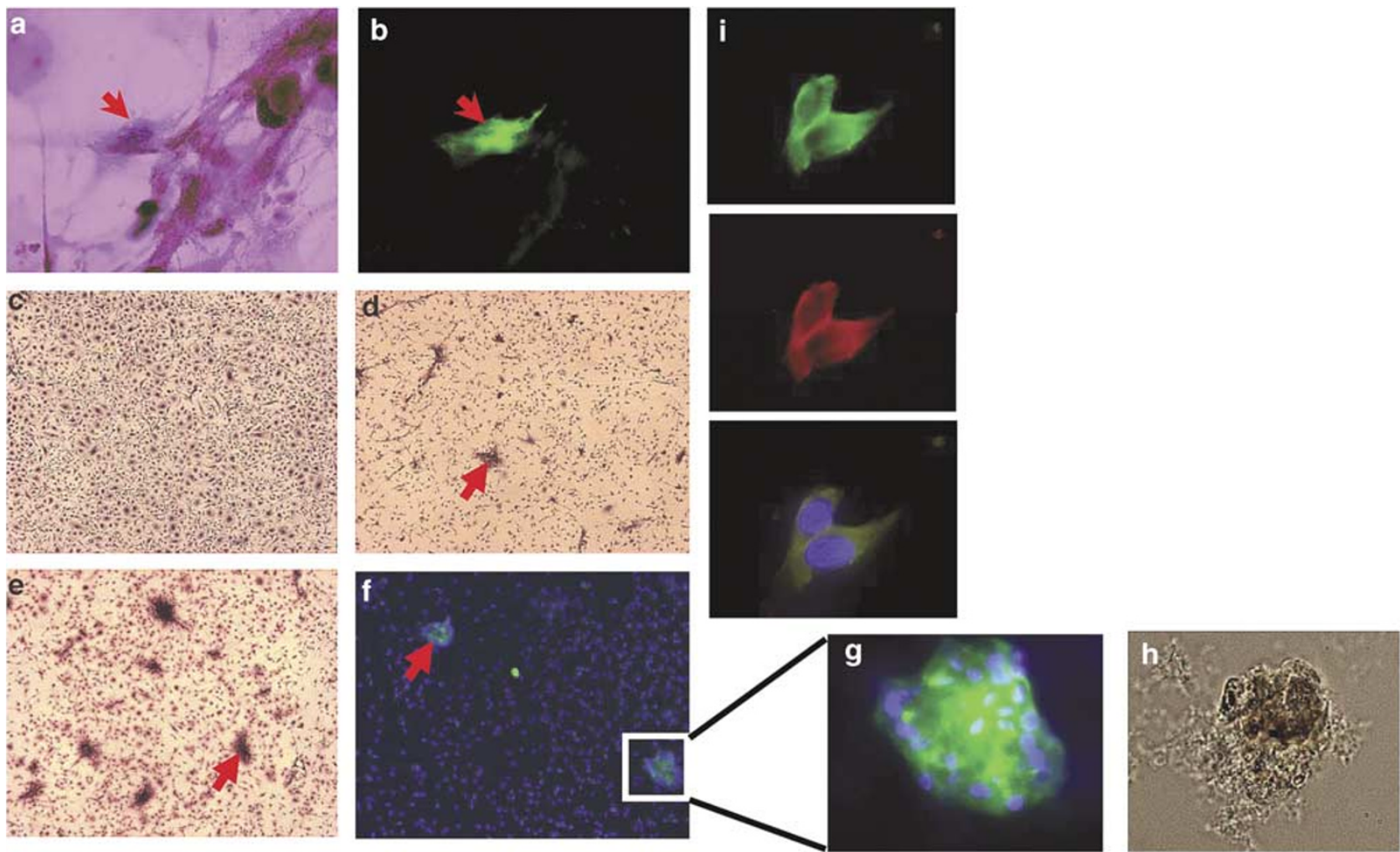

Figure 2 Development of EGFP-positive cells. (a) Hemalaun-eosin (HE) staining of adherent bone marrow stem (BMS) cells treated with retinoic acid (RA) for $24 \mathrm{~h}, \times 600$. (c) Fluorescence image of HE-stained BMS cells shows a few EGFP-positive cells (marked), indicating differentiation of BMS cells to EGFP-positive cells, $\times 600$. (c-e) HE staining of BMS cells after treatment with RA for 7, 14 and 21 days, $\times 200$. (f) From day 14, colonies of EGFP-positive cells were observed (red arrow), $\times 200$. (g) Higher magnification of one of the EGFPpositive colonies is shown, $\times 400$. (h) The GFP-positive colonies express alkaline phosphatase. (i) GFP-positive cells (green) show positive immunoreaction with antibody against Mvh (red), in lower part a merged image is shown. 
(FACS) (Figure 1a). We isolated and cultured BM cells using a procedure which supports expansion of MSC. After induction with RA (final concentration $10^{-6} \mathrm{M}$ for $24 \mathrm{~h}$ ), we identified a small population of cells $(3 \%)$ within cultured adherent BM cells that showed EGFP expression (Figures 1b, 2a and b), an indication for differentiation of BMS cells to male germ cells. In noninduced BMS cells, no expression of EGFP was observed (Figure 1c). In contrast, we detected no EGFP-positive cells within cultured fibroblast cells hematopoetic stem cells (CD45 positive) isolated from Stra8-EGFP transgenic mice after RA treatment (same conditions) (Figure 1d and e). To investigate the effects of RA treatment on BMS cell differentiation, we treated cells for various periods of time with RA: 7 days (Figure 2c), 14 days (Figure 2d) and 21 days (Figure 2e). Large colonies of cells positive for EGFP (Figure $2 \mathrm{f}$ and g) were observed that express alkaline phosphatase (Figure $2 \mathrm{~h}$ ) after 14 days of RA treatment. Using immunohistochemical analysis, Mvh was found in EGFPpositive cells (Figure 2i). After RA induction for
21 days, the positive cells were selected using FACS and designated as BMS-GC (BMS cells derived germ cells). After sorting, the cells were characterized by determining the expression of different markers for PGCs, SSCs and spermatogonia.

Immunohistochemistry and RT-PCR showed an enhanced expression of the POU domain transcription factor Oct4 in BMS-GC cells (Figures 3 and 4). Oct4 is expressed in pluripotent embryonic cells and after gastrulation, Oct4 is expressed in PGCs. ${ }^{9}$ In male mice, the expression of Oct 4 is restricted to undifferentiated spermatogonia. ${ }^{9}$ A low level of Oct4 expression was observed in untreated BMS cells (Figure 4). Oct4 is primarily expressed in pluripotent lineages and regarded to have a role in germ cell development. Furthermore, we used RT-PCR to detect expression of fragilis, stella, Mvh; and Rnf17 genes, which are expressed in early stage of male germ cell development. ${ }^{6-8,22}$ All four genes were expressed in BMS-GC, but not in untreated BMS (Figure 4). Fragilis is the first gene to mark the onset of germ cell competence, and it follows
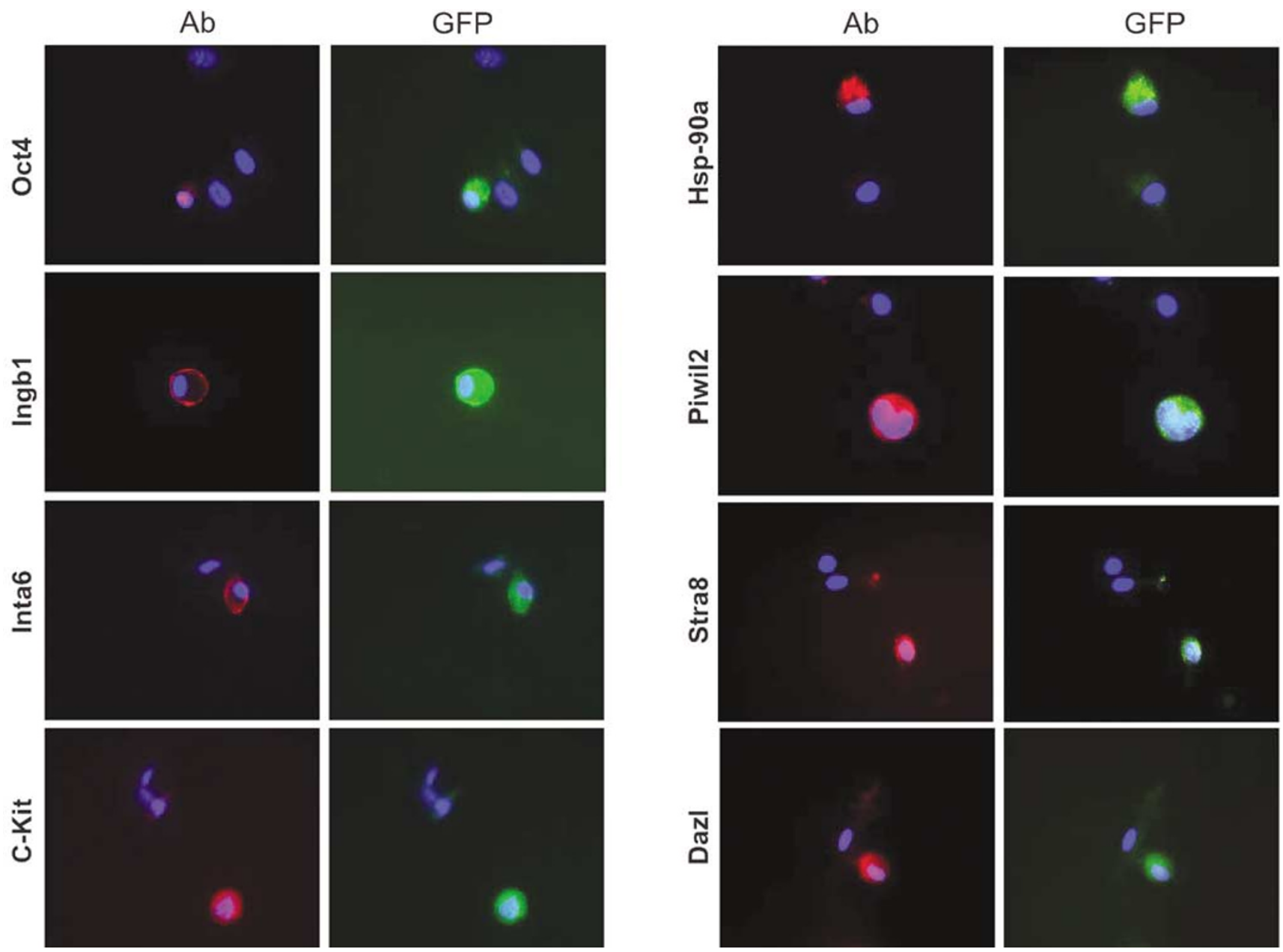

Figure 3 Immunohistochemical analysis of BMS derived EGFP-positive cells after 7 days RA treatment. Specific antibodies (Ab) against Oct4, spermatogonial stem cell markers, integrin $\beta 1$ (Ingb1) and integrin $\alpha 6$ (Inga6), spermatogonia markers, c-kit, heat-shock protein $90 \alpha$ (Hsp90 $\alpha$ ), Piwil2, Stra8 and Dazl were used (red). Nuclei are shown in blue, DAPI (4', $6^{\prime}$-diamidino-2-phenylindole) staining. Using Dazl antibody, cytoplasmic localization is demonstrated. 

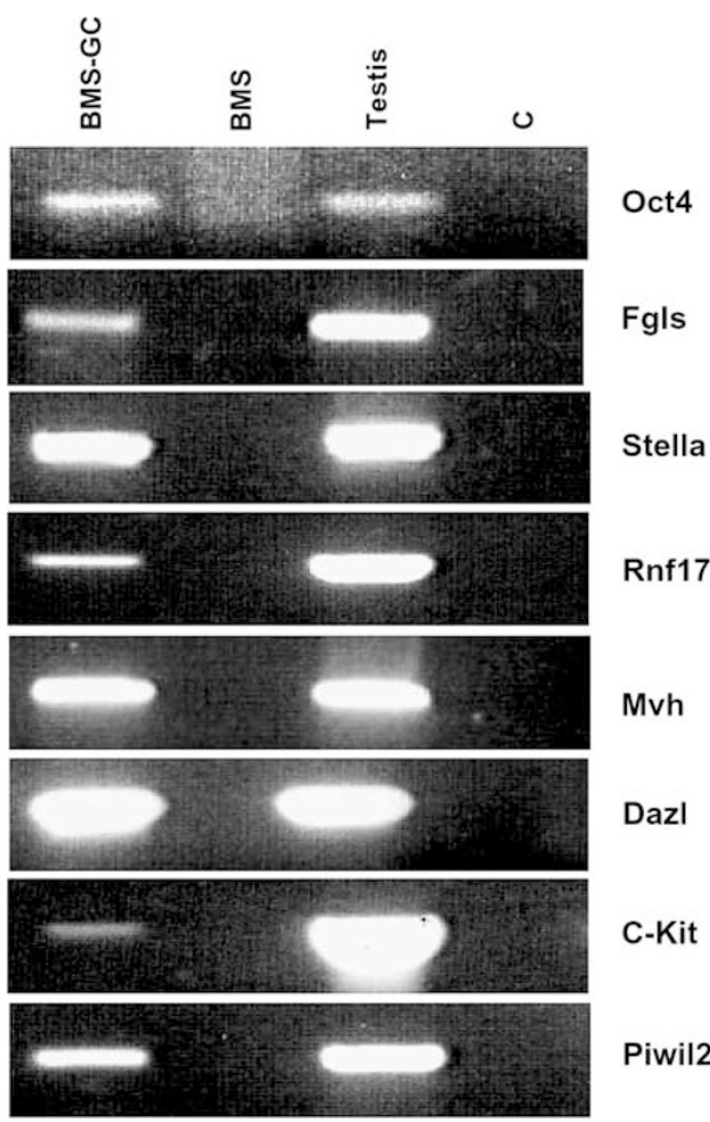

Piwil2

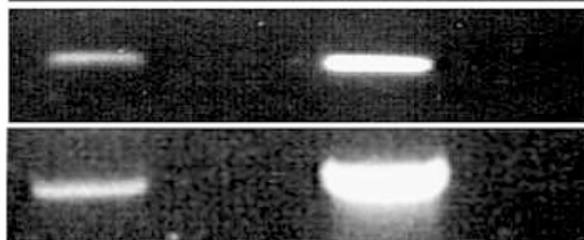

Rbm

Stra8

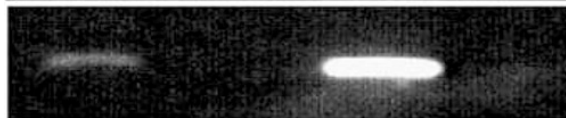

Tex18

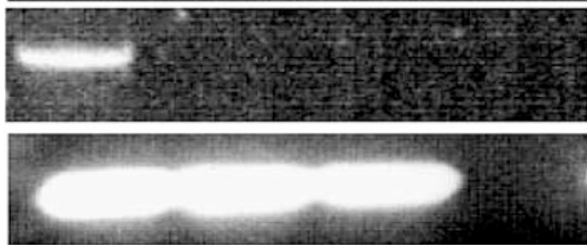

GFP

Gapdh

Figure 4 Expression analysis of BMS-GC. RNA was prepared from EGFP-sorted cells (BMS-GC), from adherent bone marrow stem cells without RA treatment (BMS) and from adult wild-type testis and processed for RT-PCR. GAPDH served as a control. C, notemplate control.

a pattern consistent with the eventual segregation of germ cells from allantoic precursors. ${ }^{6}$ It was shown that cells with the highest expression of fragilis subsequently express stella, a gene that we detected exclusively in lineage-restricted germ cells. Fragilis and stella are involved in initiating germ cell competence and specification, and in the demarcation of PGCs from their somatic neighbors. ${ }^{6} \mathrm{Mvh}$, which encodes an ATP-dependent RNA helicase, is specific for differentiating germ cells from the late migration stage to the postmeiotic stage ${ }^{8}$ Loss of Mvh function causes a deficiency in the proliferation and differentiation of male germ cells. ${ }^{22}$ These results indicate that a subpopulation of BSCderived cells can be characterized as PGCs.

Previously it was demonstrated that ES can differentiate to PGCs in vitro and these cells undergo further differentiation into functional haploid gametes. ${ }^{23}$ To investigate whether BMS-GC undergo also further differentiation in vitro, we analyzed expression of known molecular markers of spermatogonia (Dazl, Piwil2, Stra8, Rbm, Hsp90 $\alpha$, c-kit), and the $\beta 1$ - and $\alpha 6$-integrins characteristic of the stem cell fraction. BMS-GC showed expression of these markers (Figures 3 and 4). Members of the deleted in azoospermia (dazl) gene family, which are also used as markers for germ cell identification, are expressed exclusively in germ cells. ${ }^{14}$ One of the protein products of the dazl gene is expressed throughout most of the life of germ cells and is required for the development of PGCs and for the differentiation and maturation of germ cells from PGCs onwards. ${ }^{24}$ Dazl protein is present in both the nuclei and cytoplasm of fetal gonocytes and in spermatogonial nuclei. The proteins relocate to the cytoplasm during male meiosis. ${ }^{25}$ All three expression patterns, cytoplasmic, nucleic and both, were observed in BMS-GC cells. Cytoplasmic location of Dazl protein is shown in Figure 3 which is an indication for differentiation of these cells through SSCs into differentaited spermatogonia. To investigate the differentiation pathway of EGFP-positive colonies, these colonies were analyzed using confocal stereology and three-dimensional analysis. In first cell layer, EGFP-positive cells were observed (about 63\% in Figure $5 \mathrm{~h}-\mathrm{p})$ which differentiate to $\left(\mathrm{EGFP}^{+}\right.$/ $\mathrm{c}^{-} \mathrm{kit}^{+}$) in next layers (about $27 \%$ in Figure 5b-g). These cells differentiate finally to $\left(\mathrm{EGFP}^{-} / \mathrm{c}-\mathrm{kit}^{+}\right.$) in upper layers (about $10 \%$ in Figure 5a). In consideration of expression pattern of Stra8-GFP and c-kit in male germ cells, these results suggest that the first fraction $\left(\mathrm{GFP}^{+} / \mathrm{c}^{-\mathrm{kit}^{-}}\right)$correspond to prospermatogonia and SSCs, the cells expressing both GFP and c-kit $\left(\mathrm{GFP}^{+} / \mathrm{c}^{-k i t^{+}}\right)$may represent type A spermatogonia and the cells $\left(\mathrm{GFP}^{-} / \mathrm{c}-\mathrm{kit}^{+}\right)$which did not express GFP but c-kit likely represent differentiated spermatogonia (from $A_{a l}$ to $B$ spermatogonia). Presence of SSC was confiremd by expression of integrin $\alpha_{6}$ and integrin $\beta_{1}$ by immunohistochemistry, which are cell surface markers for SSC. BMS-GC cells were negative for synaptonemal complex protein $3,{ }^{26}$ phosphoglycerate kinase 2 (Pgk2), ${ }^{27}$ $\operatorname{acrosin}^{28}$ and transition protein $2,{ }^{28}$ which are markers for meiotic and postmeiotic germ cells (data not shown). This is an indication for differentiation arrest of BSC-derived germ cells at the premeiotic stages in vitro.

To investigate spermatogonial stem cell capacity and further development of BMS-GC cells in vivo, cells were transplantated into the testes of germ 

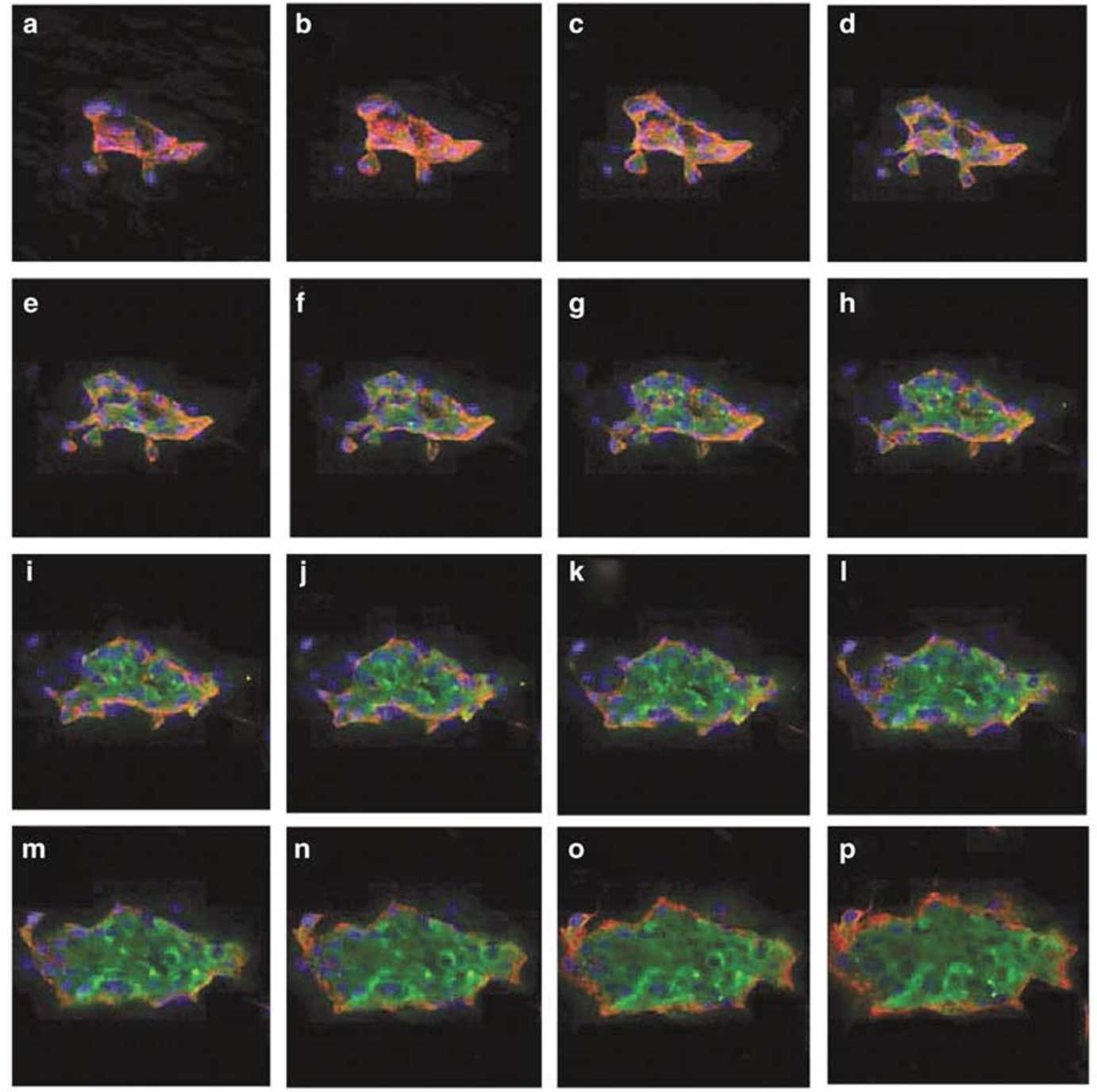

q

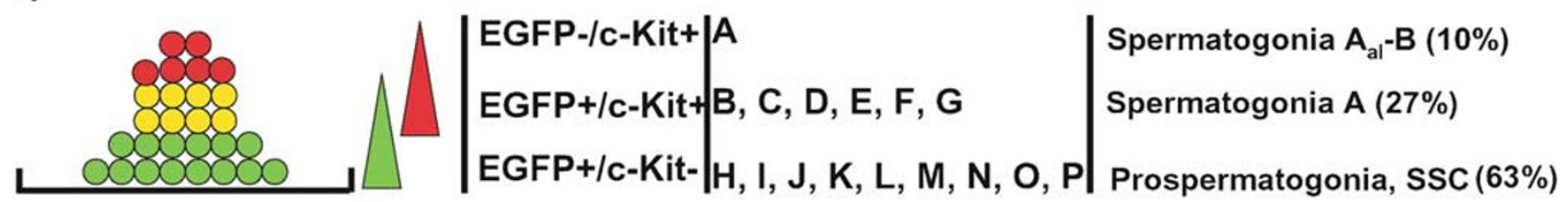

Figure 5 Confocal microscopical analysis of a EGFP-positive cell (after 21 days RA treatment) colony (green) with an immunoreactivity to c-kit antibody (red) in different cell layers beginning from bottom of cell colony show EGFP-positive cells in first cell layers (h-p) which differentiated to cell layers positive for both EGFP and c-kit (b-g) and finally to cells positive only for c-kit (a). (q) Graphical representation of differentiation pathway of cells within cell colonies. This revealed that cell colony consisted of layers of $63 \%$ EGFP + /

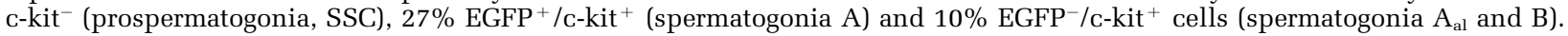

cell-depleted recipient mice. Four independent BMS-GC cultures were transplanted into the 12 busulfan-tretated mice. After 8-12 months, BMS-GC cells were able to proliferate and migrate to the basement membrane and colonize the tubules of four mice $(33.3 \%)$ (Figure 6). Cell concentration for transplantation was $5-10 \times 10^{6}$ cells $/ \mathrm{ml}$. Approxi- mately $10 \mu \mathrm{l}$ of cell suspension was transplanted. Therefore, a maximum of $10^{5}$ cells were transplanted into testis. Colonization of BMS-GC was evaluated by histological sectioning. Each slide was viewed at a magnification of $\times 400$ for the analysis. To assess proliferation and differentiation in the recipient testis, the numbers of tubule cross-sections 

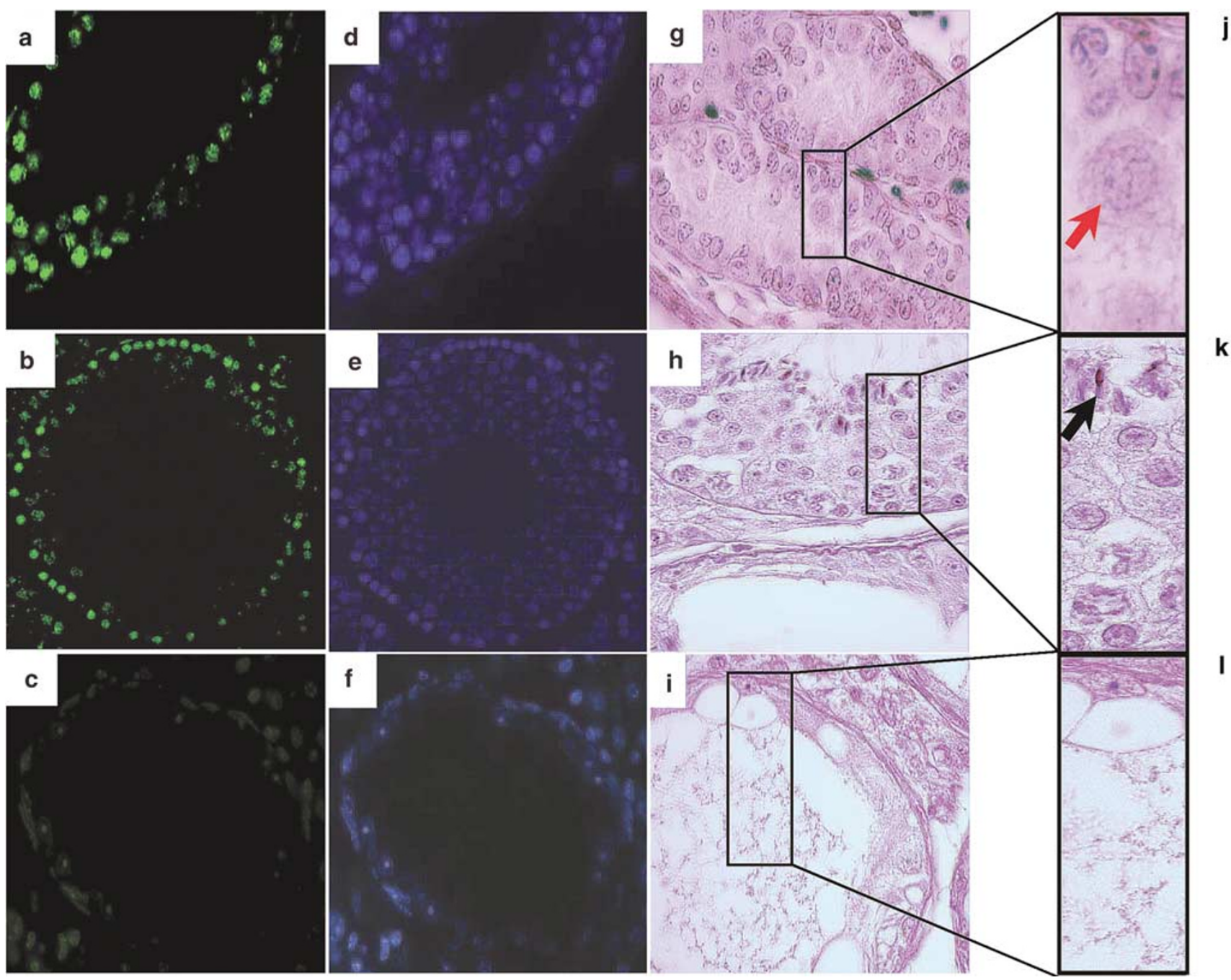

Figure 6 Analysis of BMS-GC differentiation in vivo. (a-c) Fluorescence image of a section of a transplanted testis with BMS-GC (a) and with GFP-positive cells from testis which contain spermatogonial stem cells (SSC) (b). (c) Section of a nontransplanted testis, $\times 200$. (d-f) the corresponding DAPI staining. (g-h) Hemalaun-eosin staining of a section of a testis transplanted with BMS-GC (g) with SSC (h). (i) Section of a nontransplanted testis, $\times 200$. (j-l) Higher magnification $(\times 600)$ of HE-stained section shows spermatogonial proliferation and partial differentiation (red arrow) of BMS-GC (j) and differentiation of transplanted SSC (k) to sperm (black arrow), no regeneration of spermatogenesis was observed in nontransplanted testis (l).

with evidence of proliferation and differentiation (defined as the presence of multiple layers of germ cells in the entire circumference of the seminiferous tubule) were recorded for 10 sections from each testis, and at least 55 seminiferous tubules were counted. In the sections of treated nontransplanted testis, no GFP-positive cells were observed. In transplanted testis, three to five colonies per $1 \times 10^{7} \mathrm{GFP}$ positive cells were found. This finding indicates that from $10^{5}$ transplanted cells $3-5 \times 10^{7}$ are produced. This is $3-5 \times 10^{2}$-fold over the number of transplanted cells, which is evidence for in vivo proliferation of BMS-GC after transplantation. As shown in Figure 6b, GFP-positive cells differentiated to GFP-negative cells in vivo. It is known that Stra8 is expressed in spermatogonia. Differetiation of GFP-positive cells to GFP-negative cells after transplantation are an indication for in vivo differentiation of GFP-positive BMS-GC cells.

Although we observed a proliferation of EGFPpositive spermatogonia (Figure 6a) and in some seminiferous tubuli premeiotic spermatocytes could be seen (Figure 6a, g and j), no further differentiation was detected in vivo. Premeiotic germ cells could also be detected using immunohistological analysis of testicular section with two premeiotic antobodies against Dazl (Figure 7a) and piwil2 (Figure 7a), however, no staining was observed with antibodies against postmeiotic proteins transition protein 2 and outer acrosomal membrane protein OAM (data not shown). These results are further evidence for differentiation of BMS-GC to premeiotic germ cells and arrest of differentiation in this stage. No regeneration of spermatogenesis was observed in 
a
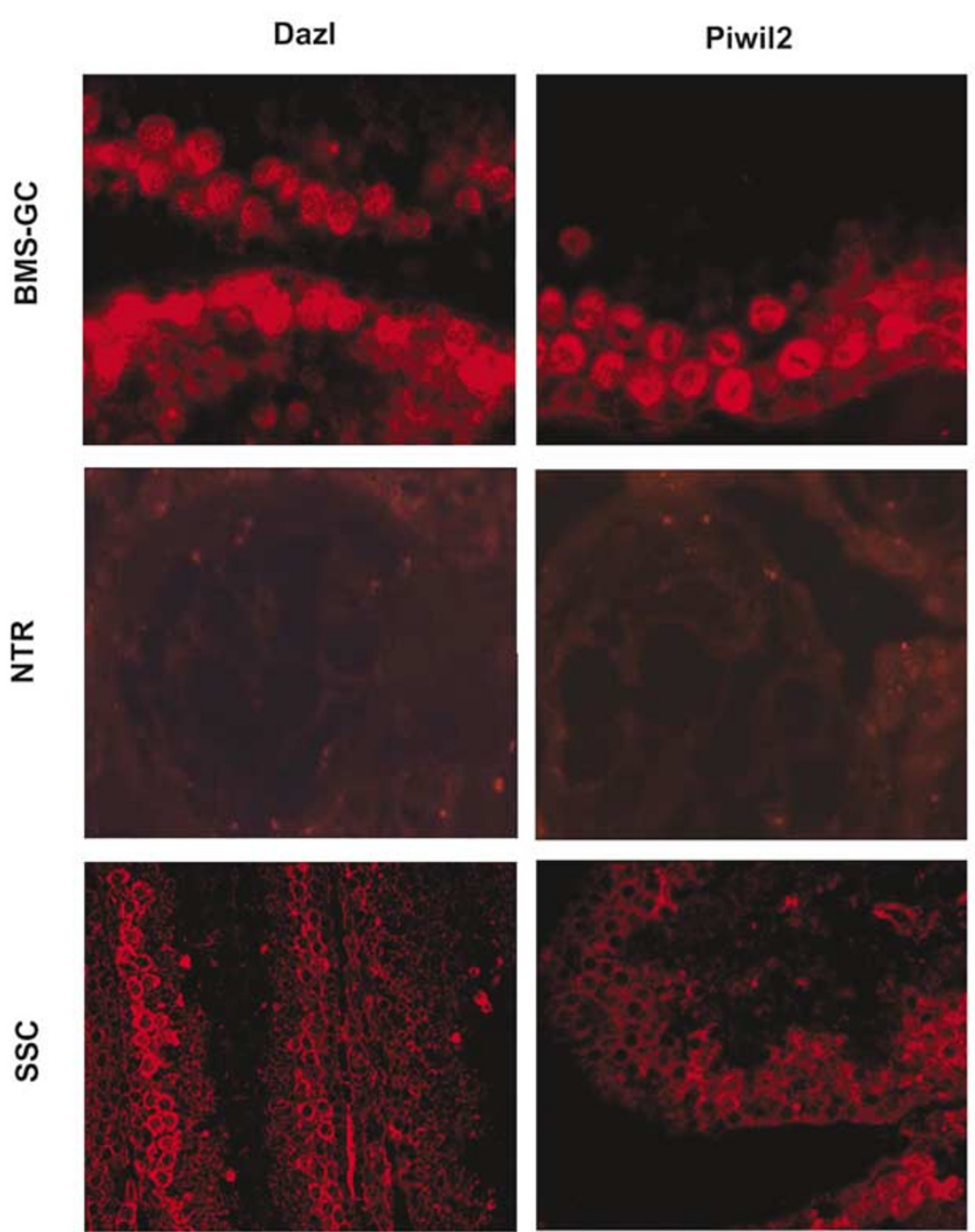

b

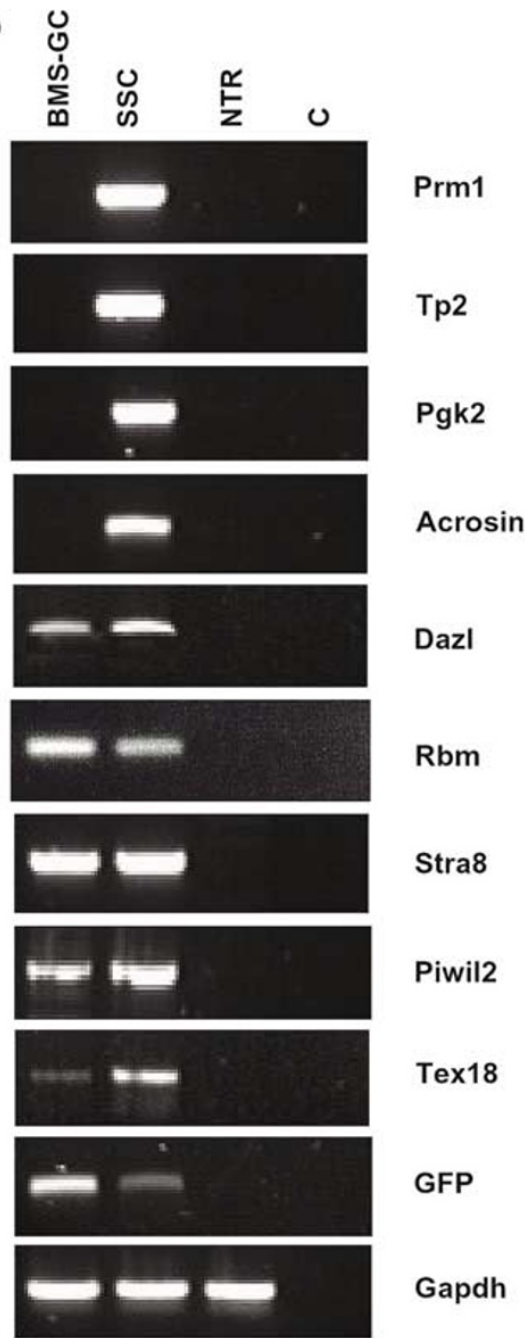

Figure 7 (a) Immunohistological analysis of section of testis transplanted with BMS-GC shows expression of germ cell-specific Dazl (red) and Piwil2 proteins. Fluorescence staining of a nontransplanted testis (NTR), no regeneration of spermatogenesis was observed. Positive staining of premeiotic cells was observed after transplantation of SSC (see Figure $6 \mathrm{~h}$ and k). (b) RT-PCR analysis of testis transplanted with BMS-GC compared with testis transplanted with testicular EGFP-positive cells (SSC) using premeiotic and postmeiotic genes. NTR, RT-PCR with RNA isolated from a nontransplanted testis. C, no-template control.

nontransplanted control testes (Figures 6c and 7a). As control, we isolated EGFP-positive cells from testis of Stra8-EGFP mouse line using FACS, which contain SSCs, and transplanted these cells into seminiferous tubuli of germ cell-depleted mice. After 8-12 months, regeneration of spermatogenesis and differentiation to sperm was found (Figures $6 \mathrm{~b}$ and $7 \mathrm{a}$ ).

Formation of the male gamete occurs in sequential mitotic, meiotic and postmeiotic phases. Many germ cell-specific transcripts are produced during this process. Their expression is developmentally regulated and stage specific. To further substantiate in vivo differentiation of BMS-GC on the molecular level, we examined expression of different stagespecific genes in transplanted testes. As shown by RT-PCR, the transplanted testes with BMS-GC cells were positive for all premeiotic genes (Tex18,
Stra8, Piwil2, Rbm and Dazl) but not positive for postmeiotic genes (acrosin, Pgk2, Tp2 and Prm1) (Figure 7b) providing an additional evidence for differentiation arrest in premeiotic stage. While no RT-PCR products for premeiotic and postmeiotic germ cells were detected in nontransplanted germ cell-depleted testes, testes transplated with SSCs showed expression of genes in all differentiation stages (Figure 7b).

\section{Discussion}

Our finding addresses one of the critically important questions in the field of stem cell plasticity. We demonstrated that a somatic adult stem cell can differentiate to male germ cells. We demonstrated that BMS cells are able to differentiate to early germ 
cells, PGCs and even SSC and spermatogonia in vitro and in vivo.

Four studies published in recent months have dramatically changed the outlook on infertility and germ cell development, as the message they delivered is that male and female gametes can be produced in vitro from embryonic or teratocarcinoma stem cells. ${ }^{21,23,29,30}$ These studies have used embryonic or teratocarcinoma stem cells to establish a population of primordial germ cell-like cells, which, either by transplantation into the testes or by extending cultures, established differentiation into male or female gametes. One of the important but unresolved questions in our study is differentiation arrest of BMS-GC at premeiotic stage which should be investigated in further studies. It indicates failure in the meiotic program of BMS-GC. No embryoid body (EB) was observed in our differentiated adherent BM cell culture which was present in differentiated ES cell cultures. Given our observation of male germ cell development, we speculate that EBs may preserve more of the tissue organization reflective of the embryonic gonadal ridge, thereby enabling male germ lineage specification. EBs support a program of male germ cell differentiation, culminating in the formation of haploid cells that manifest the morphology and fertilization potential of male haploid germ cells of the round spermatid stage. ${ }^{23}$ Microarray cDNA analysis between ES derived and BMS-GC gives more insight in differences in molecular profiling of these two types of germ cells, especially in differences in genes involved in meiosis. Furthermore, coculture of BMS-derived germ cells and EBs might also support further differentiation of BMS-GC.

Johnson et al reported that mammalian ovaries possess persisting large germline stem cells, which allegedly enable follicular renewal in adult females. ${ }^{31}$ Recently, the same research group reported that mammalian oocytes originate from putative germ cells in BM and are distributed by peripheral blood to the ovaries. ${ }^{32}$ Irrespective of their origin, BMS-GC provides important information of potential clinical applications in reproductive medicine. In contrast to ES cells, BMS-GC can be selected from autologous $\mathrm{BM}$ and might be used for therapeutical approaches in the future.

\section{Acknowledgements}

We thank S Wolf, B Sadowski, I Schwandt, D Meyer and Maren Steckel for excellent technical assistant. We thank M Schindler, H Riedesel and S Wolf for their assistance in generation of transgenic mice. We thank D Böhm for his help in Q-PCR and genotyping of transgenic mice. We thank Professor Schöler for providing of antibody against Mvh. This work was supported by the grant (Forschungsförderungsprogramm Stammzelle to $\mathrm{KN}$ of the University of Göttingen).

\section{References}

1 Lawson KA, Hage WJ. Clonal analysis of the origin of primordial germ cells in the mouse. Ciba Found Symp 1994;182:68-91.

2 McLaren A. Germ and somatic cell lineages in the developing gonad. Mol Cell Endocrinol 2000;163:3-9.

3 Donovan PJ, Stott D, Cairns LA, et al. Migratory and postmigratory mouse primordial germ cells behave differently in culture. Cell 1986;44:831-838.

4 de Rooij DG, Grootegoed JA. Spermatogonial stem cells. Curr Opin Cell Biol 1998;10:694-701.

5 Nayernia K, Li M, Engel W. Spermatogonial stem cells. In: Schatten H (ed). Germ Cell Protocols: Methods Mol Biol, Vol. 253. Humana Press Inc.: Totowa, NJ, 2003, pp 105-120.

6 Saitou M, Barton SC, Surani MA. A molecular programme for the specification of germ cell fate in mice. Nature 2002;418:293-300.

7 Wang PJ, McCarrey JR, Yang F, et al. An abundance of $\mathrm{X}$-linked genes expressed in spermatogonia. Nat Genet 2001;27:422-426.

8 Toyooka Y, Tsunekawa N, Takahashi Y, et al. Expression and intracellular localization of mouse Vasahomologue protein during germ cell development. Mech Dev 2000;93:139-149.

9 Scholer HR, Ruppert S, Suzuki N, et al. New type of POU domain in germ line-specific protein Oct-4. Nature 1990;344:435-439.

10 Elliott DJ, Ma K, Kerr SM, et al. An RBM homologue maps to the mouse Y chromosome and is expressed in germ cells. Hum Mol Genet 1996;5:869-874.

11 Vincent S, Segretain D, Nishikawa S, et al. Stagespecific expression of the Kit receptor and its ligand (KL) during male gametogenesis in the mouse: a KitKL interaction critical for meiosis. Development 1998; 125:4585-4593.

12 Oulad-Abdelghani M, Bouillet P, Decimo D, et al. Characterization of a premeiotic germ cell-specific cytoplasmic protein encoded by Stra8, a novel retinoic acid-responsive gene. J Cell Biol 1996;135:469-477.

13 Kuramochi-Miyagawa S, Kimura T, Yomogida K, et al. Two mouse piwi-related genes: miwi and mili. Mech Dev 2001;108:121-133.

14 Cooke HJ, Lee M, Kerr S, et al. A murine homologue of the human DAZ gene is autosomal and expressed only in male and female gonads. Hum Mol Genet 1996;5: 513-516.

15 van Pelt AM, Roepers-Gajadien HL, Gademan IS, et al. Establishment of cell lines with rat spermatogonial stem cell characteristics. Endocrinology 2002;143: 1845-1850.

16 Shinohara T, Avarbock MR, Brinster RL. Beta1and alpha6-integrin are surface markers on mouse spermatogonial stem cells. Proc Natl Acad Sci USA 1999;96:5504-5509.

17 Srour EF, Jetmore A, Wolber FM, et al. Homing, cell cycle kinetics and fate of transplanted hematopoietic stem cells. Leukemia 2001;15:1681-1684.

18 Young HE, Black AC. Adult stem cells. Anat Rec 2004; 276A:75-102.

19 Grove JE, Bruscia E, Krause DS. Plasticity of bone marrow-derived stem cells. Stem Cells 2004;22: 487-500.

20 Jiang Y, Jahagirdar BN, Reinhardt RL, et al. Pluripotency of mesenchymal stem cells derived from adult marrow. Nature 2002;418:41-49. 
21 Nayernia K, Li M, Jaroszynski L, et al. Stem cell based therapeutical approach of male infertility by teratocarcinoma derived germ cells. Hum Mol Genet 2004;1: 1451-1460.

22 Lacham-Kaplan O. In vivo and in vitro differentiation of male germ cells in the mouse. Reproduction 2004; 128:147-152.

23 Geijsen N, Horoschak M, Kim K, et al. Derivation of embryonic germ cells and male gametes from embryonic stem cells. Nature 2004;8:148-154.

24 Tanaka SS, Toyooka Y, Akasu R, et al. The mouse homolog of Drosophila Vasa is required for the development of male germ cells. Genes Dev 2000;14:841-853.

25 Ruggiu M, Saunders PT, Cooke HJ. Dynamic subcellular distribution of the Dazl protein is confined to primate male germ cells. J Androl 2000;21:470-477.

26 Yuan L, Liu LG, Zhao J, et al. The murine SCP3 gene is required for synaptonemal complex assembly, chromosome synapsis, and male fertility. Mol Cell 1998;5: 73-83.
27 McCarrey JR, Berg WM, Paragioudakis SJ, et al. Differential transcription of Pgk genes during spermatogenesis in the mouse. Dev Biol 1992;154:160-168.

28 Nayernia K, Adham I, Kremling H, et al. Stage and developmental specific gene expression during mammalian spermatogenesis. Int J Dev Biol 1996;40: 379-383.

29 Toyooka Y, Tsunekawa N, Akasu R, et al. Embryonic stem cells can form germ cells in vitro. Proc Natl Acad Sci USA 2003;100:11457-11462.

30 Hubner K, Fuhrmann G, Christenson LK, et al. Derivation of oocytes from mouse embryonic stem cells. Science 2003;300:1251-1256.

31 Johnson J, Canning J, Kaneko T, et al. Germline stem cells and follicular renewal in the postnatal mammalian ovary. Nature 2004;428:145-150.

32 Johnson J, Bagley J, Skaznik-Wikiel M, et al. Oocyte generation in adult mammalian ovaries by putative germ cells in bone marrow and peripheral blood. Cell 2005;122:303-315. 